PROCEEDINGS OF THE

AMERICAN MATHEMATICAL SOCIETY

Volume 129, Number 7, Pages 1885-1890

S 0002-9939(00)05881-0

Article electronically published on December 13, 2000

\title{
PUSH-FORWARD OF DEGENERACY CLASSES AND AMPLENESS
}

\author{
JØRGEN ANDERS GEERTSEN
}

(Communicated by Ron Donagi)

\begin{abstract}
Let $X$ be a projective variety and $E, F$ vector bundles on $X$. Suppose $g: X \rightarrow Y$ is a surjective map onto another variety $Y$. Let $\phi: E \rightarrow F$ be any vector bundle map and $X_{k}(\phi)$ the $k$ 'th degeneracy locus of $\phi$. We show that the dimension of $g\left(X_{k}(\phi)\right)$ is at least equal to $\min \{\operatorname{dim} Y, \operatorname{dim} X-(\operatorname{rank} E-k)(\operatorname{rank} F-k)\}$

under the hypothesis that $E^{*} \otimes F$ is an ample vector bundle on $X$.
\end{abstract}

\section{INTRODUCTION}

Let $X$ be an algebraic variety over an algebraically closed field of arbitrary characteristic and $E, F$ vector bundles on $X$, of ranks $e, f$ respectively. For any vector bundle map $\phi: E \rightarrow F$ and $k \in \mathbb{N}, 0 \leq k \leq \min \{e, f\}$, the $k$ 'th degeneracy locus of $\phi$ is the closed subset of $X$ defined by

$$
X_{k}(\phi):=\{x \in X \mid \operatorname{rank} \phi(x) \leq k\} .
$$

Degeneracy loci are studied because they turn up in a number of geometric constructions. For instance, in Brill-Noether theory, they show up as the subsets of the Jacobian that parametrizes complete linear systems of a specific degree and dimension bounded below (cf. for instance [ACGH] or [Fu-La]).

The expected dimension of $X_{k}(\phi)$ is the number $\operatorname{dim} X-(e-k)(f-k)$, which we denote by $m_{k}$. If $X_{k}(\phi)$ happens to be non-empty, its dimension is at least equal to the expected dimension. A classic result on degeneracy loci is the theorem of Fulton-Lazarsfeld [Fu-La] : Let $X$ be a projective variety and $E, F$ vector bundles on $X$ such that $E^{*} \otimes F$ is an ample vector bundle. Then for any map $\phi: E \rightarrow F$, if the expected dimension $m_{k}$ is non-negative, then $X_{k}(\phi) \neq \emptyset$. Moreover, if the expected dimension is positive, then $X_{k}(\phi)$ is connected.

We are interested in the following relative situation: $X, Y$ are projective varieties and $g: X \rightarrow Y$ a surjective map. We suppose $E^{*} \otimes F$ is an ample vector bundle. An important special case of a more general theorem of Steffen $[\mathrm{St}$ is the following: if the ground field is $\mathbb{C}$, and $\phi: E \rightarrow F$ is a map such that $X_{k}(\phi)$ is non-empty, then the dimension of $g\left(X_{k}(\phi)\right)$ is at least equal to $\min \left\{\operatorname{dim} Y, m_{k}\right\}$. Here we show how one can obtain this result easily (in arbitrary characteristic) using intersection theory. Specifically, for a map $\phi: E \rightarrow F$ as above, we consider the so-called

Received by the editors September 7, 1998 and, in revised form, October 15, 1999.

2000 Mathematics Subject Classification. Primary 14C17; Secondary 14M12.

(C)2000 American Mathematical Society 
degeneracy class $\mathbb{X}_{k}(\phi) \in A_{m_{k}}\left(X_{k}(\phi)\right)$ of $\phi$. This should be viewed as a sort of "idealized" degeneracy locus. (For the precise definition see $\S 1$.) Then we show

Theorem 1. Let $X$ be a projective variety and $E, F$ vector bundles on $X$ such that $E^{*} \otimes F$ is an ample vector bundle. Suppose $g: X \rightarrow Y$ is a surjective map onto another variety $Y$. Let $k$ be such that $0 \leq m_{k} \leq \operatorname{dim} Y$. Then for any map $\phi: E \rightarrow F$ we have $g_{*} \mathbb{X}_{k}(\phi) \neq 0$.

Taking $E=\mathcal{O}_{X}$ and $k=0$ in Theorem 1 (i.e. considering zero-sets of sections of the ample bundle $F$ ), the statement is the following non-vanishing result on top Chern classes:

Theorem 1'. Suppose $g: X \rightarrow Y$ is a surjective map of projective varieties and $F$ an ample vector bundle on $X$ of rank $f$. Suppose $0 \leq \operatorname{dim} X-f \leq \operatorname{dim} Y$. Then $g_{*} c_{f}(F) \neq 0$.

By construction, $\mathbb{X}_{k}(\phi)$ is a class supported on the actual degeneracy locus $X_{k}(\phi)$. Hence if $g_{*} \mathbb{X}_{k}(\phi) \in A_{m_{k}}\left(g\left(X_{k}(\phi)\right)\right)$ is non-zero, the dimension of $g\left(X_{k}(\phi)\right)$ must be at least equal to $m_{k}$. Therefore Theorem 1 implies the

Corollary. Let $X$ be a projective variety over a field of arbitrary characteristic and $E, F$ vector bundles on $X$ of ranks e, $f$ respectively. Suppose that $E^{*} \otimes F$ is an ample vector bundle. Let $g: X \rightarrow Y$ be a surjective map. Then for any map $\phi: E \rightarrow F$ we have $\operatorname{dim} g\left(X_{k}(\phi)\right) \geq \min \{\operatorname{dim} Y, \operatorname{dim} X-(e-k)(f-k)\}$.

In particular, taking $Y=X$ and $g=1$ in the corollary one recovers the existence result of Fulton and Lazarsfeld.

\section{Degeneracy Classes}

In this section we recall the intersection theoretic construction of the so-called local top Chern classes and more general degeneracy classes corresponding to a vector bundle map. For details, cf. [Fu, Chap. 14].

Let $E$ be a vector bundle of rank $e$ on a purely $n$-dimensional scheme $X$ and suppose $s: X \hookrightarrow E$ is a section. Denote by $0: X \hookrightarrow E$ the zero section embedding of $X$ in $E$. Consider the fibre square

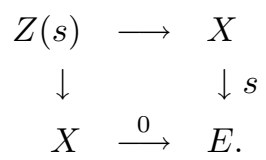

Then $0 ![X] \in A_{n-e}(Z(s))$ is called the localized top Chern class of $E$ with respect to $s$, and is denoted $\mathbb{Z}(s)$. The reason for this name is that if we denote by $i: Z(s) \hookrightarrow X$ the inclusion, then in fact

$$
i_{*} \mathbb{Z}(s)=c_{e}(E) \cap[X] \in A_{n-e}(X) .
$$

This follows directly from the basic properties of the intersection product. Therefore $\mathbb{Z}(s)$ really is a "local top Chern class" of $E$, situated on the actual zero-set $Z(s)$.

Example. The localized top Chern classes of $\mathcal{O}_{\mathbb{P}^{2}}(1)$ are just the straight lines $\subset \mathbb{P}^{2}$. In general, the localized top Chern classes of $\mathcal{O}_{\mathbb{P}^{2}}(d)$ are the (reducible) curves of degree $d$ in $\mathbb{P}^{2}$. 
Now let $E, F$ be vector bundles on $X$, of ranks $e, f$ respectively. Put $k^{\prime}=e-k$ and consider $\mathbb{G}:=G_{k^{\prime}}(E)$, the Grassmanian bundle of $k^{\prime}$-planes in $E$ with its natural projection map $\pi: G_{k^{\prime}}(E) \longrightarrow X$. On $\mathbb{G}$ we have the tautological exact sequence

$$
0 \longrightarrow \mathcal{S} \longrightarrow \pi^{*} E \longrightarrow \mathcal{Q} \longrightarrow 0
$$

where $\mathcal{S}, Q$ are the tautological sub- and quotient bundles respectively. Denote a point in $\mathbb{G}$ by $\left(x, H_{x}\right) ; H_{x}$ is a $k^{\prime}$-plane in the vector space $E(x)$. Suppose we are given a map $\phi: E \rightarrow F$ of the bundles on $X$. Consider the composition

$$
\sigma: \mathcal{S} \hookrightarrow \pi^{*} E \stackrel{\pi^{*} \phi}{\longrightarrow} \pi^{*} F .
$$

Then $Z(\sigma)=\left\{\left(x, H_{x}\right) \mid H_{x} \subset \operatorname{ker} \phi(x)\right\}$. Therefore $Z(\sigma)$ maps onto $X_{k}(\phi)$ by $\pi$, because the map $\phi(x): E(x) \rightarrow F(x)$ has rank $\leq k$ if and only if the kernel is at least $k^{\prime}$-dimensional, i.e. contains some $k^{\prime}$-plane. Letting $\bar{\pi}: Z(\sigma) \longrightarrow X_{k}(\phi)$ denote the restriction of $\pi$ to $Z(\sigma)$ we define the degeneracy class $\mathbb{X}_{k}(\phi)$ by

$$
\mathbb{X}_{k}(\phi):=\bar{\pi}_{*} \mathbb{Z}(\sigma) \in A_{m_{k}}\left(X_{k}(\phi)\right),
$$

where as usual $m_{k}=\operatorname{dim} X-(e-k)(f-k)$. Let $i$ denote the inclusion $X_{k}(\phi) \hookrightarrow X$. Recall next the expression of $i_{*} \mathbb{X}_{k}(\phi)$ in terms of the Chern classes of $E$ and $F$ :

Porteous' formula ([Fu, Th. 14.4]). We have

$$
i_{*} \mathbb{X}_{k}(\phi)=\Delta_{f-k}^{(e-k)}(c(F-E)) \cap[X] \in A_{m_{k}}(X),
$$

where $\Delta_{q}^{(p)}(c)$ denotes the determinant of the $p$ by $p$ matrix $\left(c_{q+j-i}\right)_{1 \leq i, j \leq p}$ and $c(F-E)=c(F) / c(E)$. We note that if $X_{k}(\phi)$ has the expected dimension $m_{k}$ and $X$ is Cohen Macaulay, then in fact $\left[X_{k}(\phi)\right]=\mathbb{X}_{k}(\phi)$ so in this case one gets a formula for the fundamental class of $X_{k}(\phi)$.

We will apply the construction of the degeneracy classes to the situation at hand in the following way. Suppose $g: X \rightarrow Y$ is a proper surjective map and $\phi: E \rightarrow F$ a morphism. Suppose we could show that $g_{*} \mathbb{X}_{k}(\phi) \neq 0$ in $A_{m_{k}}(Y)$. Then since $\mathbb{X}_{k}(\phi)$ is constructed on $X_{k}(\phi), g_{*} \mathbb{X}_{k}(\phi)$ is actually a cycle class in $A_{m_{k}}\left(g\left(X_{k}(\phi)\right)\right)$. In particular $A_{m_{k}}\left(g\left(X_{k}(\phi)\right)\right) \neq 0$; but then certainly $\operatorname{dim} g\left(X_{k}(\phi)\right) \geq m_{k}$ and we are done.

\section{Push-Forward of Degeneracy Classes}

Before we prove Theorem 1 we make the following observation.

Lemma 2.1. Suppose $X$ is a projective variety and $g: X \rightarrow \mathbb{P}^{m}$ a surjective map. Then for each $0 \leq l \leq m$, there is an l-plane $H \subset \mathbb{P}^{m}$ such that all irreducible components of $g^{-1}(H)$ have dimension $\operatorname{dim} X-m+l$.

Proof. Let $\mathbb{G}=G_{l}\left(\mathbb{P}^{m}\right)$ be the Grassmanian of $l$-planes in $\mathbb{P}^{m}$. Consider the incidence correspondence

$$
Q:=\{(x, H) \mid g(x) \in H\} \subset X \times \mathbb{G} .
$$

First we claim that $Q$ is irreducible, of dimension $\operatorname{dim} X+l(m-l)$. To see this look at the projection map $p_{1}: Q \rightarrow X$. Then for any $x \in X$,

$$
\begin{aligned}
p_{1}^{-1}(x) & \cong\{H \in \mathbb{G} \mid g(x) \in H\} \\
& \cong G_{l-1}\left(\mathbb{P}^{m-1}\right)
\end{aligned}
$$


is an irreducible subvariety of dimension $l(m-l)$. By [Hs, Th. 11.14], this proves the claim.

Next consider the second projection map $p_{2}: Q \rightarrow \mathbb{G}$. This map is surjective since any $l$-plane contains some image point of $g$ ( $g$ is surjective !). Hence by $[\mathrm{Mu}$. I.8] the generic fiber consists of components of dimension equal to

$$
\begin{aligned}
\operatorname{dim} Q-\operatorname{dim} \mathbb{G} & =\operatorname{dim} X+l(m-l)-\operatorname{dim} \mathbb{G} \\
& =\operatorname{dim} X+l(m-l)-(l+1)(m-l) \\
& =\operatorname{dim} X-m+l .
\end{aligned}
$$

However, for any $H \in \mathbb{G}, p_{2}^{-1}(H) \cong g^{-1}(H)$, so we are done.

Remark. Not surprisingly, the proof shows that there is in fact an open set $U \subset \mathbb{G}$ of $l$-planes satisfying the conclusion of the lemma.

Next recall the following theorem of Fulton and Lazarsfeld (cf. [Fu, Th. 12.1]).

Theorem 2.2. Suppose $E$ is an ample vector bundle of rank $e$ on the projective variety $X$. Let $V \subset E$ be a subvariety of dimension $d \geq e$ and let $s: X \hookrightarrow E$ be a section of $E$. Then for any ample line bundle $\mathcal{L}$ on $X$,

$$
\int_{X} c_{1}(\mathcal{L})^{d-e} \cap s^{*}[V]>0 .
$$

We just need the special case where $d=e$ in Theorem 2.2. Now we prove

Theorem 2.3. Let $X$ be a projective variety and $E, F$ vector bundles on $X$ of ranks e, $f$ respectively, and assume that $E^{*} \otimes F$ is an ample vector bundle. Suppose $g: X \rightarrow Y$ is a surjective map onto another variety $Y$. Let $k$ be such that $0 \leq$ $m_{k} \leq \operatorname{dim} Y$. Then for any map $\phi: E \rightarrow F$ we have $g_{*} \mathbb{X}_{k}(\phi) \neq 0$.

Proof. Since any projective variety admits a finite map onto some projective space, we might as well assume that $Y=\mathbb{P}^{m}$. Let $l=m-m_{k}$. Then by assumption, $0 \leq l \leq m$ so Lemma 2.1 gives a linear subspace $H \subset \mathbb{P}^{m}$ of dimension $l$ such that $W:=g^{-1}(H)$ is equidimensional, of dimension $=\operatorname{dim} X-m_{k}=(e-k)(f-k)$, and

$$
c_{1}\left(g^{*} \mathcal{O}(1)\right)^{m_{k}} \cap[X]=[W] .
$$

By the projection formula,

$$
\int_{\mathbb{P} m} c_{1}(\mathcal{O}(1))^{m_{k}} \cap g_{*} \mathbb{X}_{k}(\phi)=\int_{X} c_{1}\left(g^{*} \mathcal{O}(1)\right)^{m_{k}} \cap \mathbb{X}_{k}(\phi) .
$$

Now $W$, being the preimage of the complete intersection $H$, is certainly a local complete intersection. If $X$ was Cohen Macaulay, then the embedding $W \hookrightarrow X$ would therefore be regular. Then one could apply the general fact that the formation of degeneracy classes commutes with Gysin morphisms to arrive at a situation where Theorem 2.2 applies.

However, we can avoid assuming that $X$ is Cohen Macaulay by using Porteous' formula. Letting $i: X_{k}(\phi) \hookrightarrow X$ denote the inclusion, this reads

$$
i_{*} \mathbb{X}_{k}(\phi)=\Delta_{f-k}^{(e-k)}(c(F-E)) \cap[X] \in A_{m_{k}}(X) .
$$

We won't worry about the explicit formula; the important thing to notice is that the formula expresses (the image of) $\mathbb{X}_{k}(\phi)$ in terms of certain Chern classes of $E$ 
and $F$. Plugging this into the above equation and using $(*)$ and the projection formula on the inclusion $W \hookrightarrow X$ gives

$$
\begin{aligned}
\int_{X} c_{1}\left(g^{*} \mathcal{O}(1)\right)^{m_{k}} \cap \mathbb{X}_{k}(\phi) & =\int_{X} c_{1}\left(g^{*} \mathcal{O}(1)\right)^{m_{k}} \cap \Delta_{f-k}^{(e-k)}(c(F-E)) \cap[X] \\
& =\int_{X} \Delta_{f-k}^{(e-k)}(c(F-E)) \cap[W] \\
& =\int_{W} \Delta_{f-k}^{(e-k)}\left(c\left(F_{W}-E_{W}\right)\right),
\end{aligned}
$$

where $E_{W}, F_{W}$ denotes the restriction of $E, F$ to $W$. If we denote by $\mathbb{W}_{k}\left(\phi^{\prime}\right) \in$ $A_{0}(W)$ the image in $A_{0}(W)$ of the degeneracy class of the restriction $\phi^{\prime}$ of $\phi$ to $W$, then reading the Porteous formula backwards gives

$$
\int_{W} \Delta_{f-k}^{(e-k)}\left(c\left(F_{W}-E_{W}\right)\right)=\int_{W} \mathbb{W}_{k}\left(\phi^{\prime}\right) .
$$

Hence

$$
\int_{\mathbb{P} m} c_{1}(\mathcal{O}(1))^{m_{k}} \cap g_{*} \mathbb{X}_{k}(\phi)=\int_{W} \mathbb{W}_{k}\left(\phi^{\prime}\right) .
$$

As remarked above, one could arrive at this expression quicker if $X$ was Cohen Macaulay. However, the Porteous formula saves us assuming that, and now it has played its role. All we have to do now is to show that the cycle class $\mathbb{W}_{k}\left(\phi^{\prime}\right)$ has positive degree. To this end we use another description of the degeneracy class. Let $\pi: E_{W}^{*} \otimes F_{W} \rightarrow W$ be the projection map. Then on $E_{W}^{*} \otimes F_{W}$ there is a tautological map

$$
\begin{aligned}
& \tau: \pi^{*} E_{W} \longrightarrow \pi^{*} F_{W} \\
& \tau(x, h): E(x) \stackrel{h}{\longrightarrow} F(x) .
\end{aligned}
$$

The $k^{\prime}$ th degeneracy locus $D_{k}(\tau)$ of $\tau$ is pure $e f$-dimensional, and

$$
\mathbb{W}_{k}\left(\phi^{\prime}\right)=\phi^{\prime *}\left[D_{k}(\tau)\right],
$$

where $\phi^{\prime *}$ is the Gysin map associated to the section $\phi^{\prime}: W \rightarrow E_{W}^{*} \otimes F_{W}$ (cf. remark following Th. $14.4 \mathrm{in}[\mathrm{Fu}]$ ). By assumption $E_{W}^{*} \otimes F_{W}$ is ample; hence $\phi^{\prime *}\left[D_{k}(\tau)\right] \in A_{0}(W)$ has strictly positive degree by Theorem 2.2. This concludes the proof of Theorem 2.3.

Remark. In the proof of Theorem 2.3 we use much less than the amplitude of $E^{*} \otimes F$ on $X$. In fact, we only use that there is some $H \subset \mathbb{P}^{m}$ such that $g^{-1}(H)$ has the 'right' dimension and the restriction of $E^{*} \otimes F$ to $g^{-1}(H)$ is ample.

If one takes $E=\mathcal{O}_{X}$ and $k=0$, which amounts to considering zero-schemes of sections of the bundle $F$, then the degeneracy class $\mathbb{X}_{k}(\phi)$ is simply the localized top Chern class $\mathbb{Z}(\phi)$ of the section $\phi \in \Gamma(X, F)$, and Porteous' formula is just $i_{*} \mathbb{Z}(\phi)=c_{f}(F) \cap[X]$. Hence Theorem 2.3 becomes

Theorem 2.4. Suppose $g: X \rightarrow Y$ is a surjective map of projective varieties and $F$ an ample vector bundle on $X$ of rank $f$. Suppose $0 \leq \operatorname{dim} X-f \leq \operatorname{dim} Y$. Then $g_{*} c_{f}(F) \neq 0$. 
Finally, we have

Corollary 2.5. Let $X$ be a projective variety over a field of arbitrary characteristic and $E, F$ vector bundles on $X$ of ranks e, $f$. Suppose that $E^{*} \otimes F$ is an ample vector bundle. Let $g: X \rightarrow Y$ be a surjective map. Then for any map $\phi: E \rightarrow F$ we have $\operatorname{dim} g\left(X_{k}(\phi)\right) \geq \min \{\operatorname{dim} Y, \operatorname{dim} X-(e-k)(f-k)\}$.

Proof. Let $m_{k}=\operatorname{dim} X-(e-k)(f-k)$. If $0 \leq m_{k} \leq \operatorname{dim} Y$, then by Theorem 2.3, $0 \neq g_{*} \mathbb{X}_{k}(\phi) \in A_{m_{k}}\left(g\left(X_{k}(\phi)\right)\right)$. Hence $\operatorname{dim} g\left(X_{k}(\phi)\right) \geq m_{k}$. If $m_{k}>\operatorname{dim} Y$, then for any $y \in Y$, the dimension of the fiber $g^{-1}(y)$ is at least equal to $\operatorname{dim} X-\operatorname{dim} Y>$ $(e-k)(f-k)$. But $\left.E^{*} \otimes F\right|_{g^{-1}(y)}$ is an ample vector bundle, so the restriction of $\phi$ to $g^{-1}(y)$ must drop rank according to the theorem of Fulton and Lazarsfeld Fu-La, Th. 1.1]. That is, $X_{k}(\phi) \cap g^{-1}(y) \neq \emptyset$. Since $y$ was arbitrary, we conclude that $X_{k}(\phi)$ surjects onto $Y$. Finally if $m_{k}<0$ the statement is trivial.

\section{ACKNOWLEDGEMENTS}

I am grateful to R. Lazarsfeld for valuable conversation.

\section{REFERENCES}

[ACGH] Arbarello, E., Cornalba, M., Griffiths, P., Harris, J.: Geometry of algebraic curves I. Springer-Verlag, Heidelberg (1985). MR 86h:14019

[Fu] Fulton, W.: Intersection Theory. Ergebnisse der Mathematik und ihre Grenzgebiete 2 (1984). MR 85k:14004

[Fu-La] Fulton, W., Lazarsfeld, R.: On the Connectedness of Degeneracy Loci and Special Divisors. Acta Math. 146 (1981), 271-283. MR 82k:14016

[Hs] Harris, J.: Algebraic Geometry. Graduate Texts in Math. 133, Springer-Verlag, Heidelberg (1992). MR 93j:14001

[Mu Mumford, D.: The Red Book of Varieties and Schemes. Lecture Notes in Math. 1358, Springer-Verlag, Heidelberg (1988). MR 89k:14001

[St] Steffen, F.: A Generalized Principal Ideal Theorem with an Application to Brill-Noether Theory. Invent. Math. 132 Fasc. 1 (1998), 73-90. MR 93c:14035

Department of Mathematics, Sproul Hall, University of California, Riverside, CalIFORNIA 92521

E-mail address: geertsen@math.ucr.edu 